\title{
Management of lower respiratory tract infections by French general practitioners: the AIR II study
}

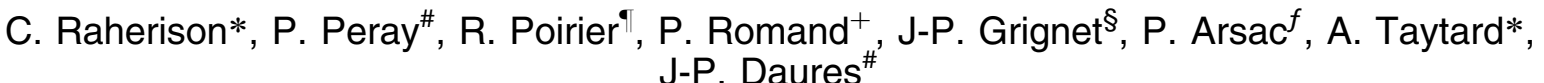

Management of lower respiratory tract infections by French general practitioners: the AIR II study. C. Raherison, P. Peray, R. Poirier, P. Romand, J-P. Grignet, P. Arsac, A. Taytard, J-P. Daures. C ERS Journals Ltd 2002.

ABSTRACT: The Analyse Infections Respiratoires (AIR) II study is a prospective, multicentre survey of the management of lower respiratory tract infections in patients aged 15-65 yrs by general practitioners (GPs) in France.

To obtain real-time data recording, practitioners were required to submit an anonymous copy of their drug prescriptions. They were then interviewed over the telephone about the patients' sociodemographic data, signs and symptoms, as well as their presumptive diagnosis and the investigations they had decided upon.

GPs $(n=3,144)$ reported 5,469 evaluable cases. Pneumonia accounted for $9.6 \%$ of diagnoses, acute exacerbations of chronic bronchitis $14.9 \%$ and acute bronchitis $72.5 \%$. The symptomatology covered an extremely wide range of clinical features, which, although statistically different in terms of incidence, overlapped to a large extent across diagnoses. By contrast, hospitalization, investigations or referral to a specialist were much more prevalent in pneumonia, although still very infrequent in general terms $(0.5$, 1.2 and $10.8 \%$, respectively). Antibiotics were prescribed in $96.5 \%$ of patients, with minor differences between diagnoses. However, other medications such as nonsteroid, anti-inflammatory drugs, steroids, nonspecific antitussives and bronchial liquefiers accounted for two-thirds of the prescriptions.

This study demonstrates the lower respiratory tract infections encountered by general practitioners are usually mild. However, antibiotic prescription was more systematic than in previous studies and the prescription of nonspecific symptomatic treatments was twice as frequent. General practitioners did not perform additional examinations or refer on a regular basis. There was a high prescription rate for symptomatic treatment.

Eur Respir J 2002; 19: 314-319.

\begin{abstract}
*Hôpital du Haut Lévêque, Bordeaux, \#Institut Universitaire de Recherche Clinique, Montpellier, ${ }^{\top}$ Centre Hospitalier du Pays d'Aix, Aix en Provence, ${ }^{+}$Centre Hospitalier le Morillon, Thonon les Bains, ${ }^{\S}$ Centre Hospitalier de Denain, Denain, ${ }^{f}$ Hopital d'Orléans, Orléans, France.
\end{abstract}

Correspondence: A. Taytard, Centre François Magendie, Hôpital du Haut Lévêque, 5 avenue de Magellan, 33604 Pessac, France.

Fax: 33556556547

E-mail: andre.taytard@chu-bordeaux.fr

Keywords: Acute bronchitis, antibiotics, chronic obstructive pulmonary disease exacerbation, lower respiratory tract illness, pharmaco-epidemiology, pneumonia

Received: February 262001

Accepted after revision August 22001

This study was sponsored by the Société de Pneumologie de Langue Française and the Société de Pathologie Infectieuse de Langue Française, and was supported by Laboratoire Aventis Pharma France.
Antibiotic prescription is associated with a very low individual risk, but, because it is common practice, it is likely to contribute to the increasing incidence of resistant bacterial strains. Thus, there is a proven direct relationship between outpatient antibiotic consumption and bacterial resistance [1], while specific efforts to restrict the prescription of the class of antibiotic have shown this relationship to be reversible [2].

Community-acquired lower respiratory tract infections (LRTI) are the leading cause of antibiotic prescriptions in France [3]. This has led to the publication of guidelines and subsequently recommendations are enforceable with respect to French practitioners. Guidelines and recommendations are intended to reduce the total volume of prescriptions, to preserve certain classes of antibiotic by a more selective choice of indications, and to encourage the use of appropriate doses to restrict their capacity for selecting resistant bacterial strains [4, 5]. Studies subsequent to these recommendations have shown that their effect is still inadequate [6, 7]. Epidemiological studies designed to evaluate the incidence of each LRTI subtype (acute bronchitis (AB), acute exacerbation of chronic bronchitis (AECB) and pneumonia) and to measure the frequency of prescriptions of the different classes of antibiotics, have often been conducted separately. These studies show the gap between guidelines and practice but do not provide an understanding of the causes.

The definition of interventional methods that are likely to have a greater impact on prescriptions requires a more detailed identification of practice in general medicine. Many factors are likely to intervene in the medical decision-making process [8] and the experts' recommendations have concerned only some of these.

The aim of the Analyse Infections Respiratoires (AIR) II study was to combine epidemiological and pharmaco-epidemiological data prospectively, in a single survey, while at the same time examining the diagnostic process in consultations together with 
social parameters. By taking into account the direct real-time recording of supplementary therapeutic and diagnostic prescriptions by general practitioners (GPs) confronted with an LRTI, it attempts to avoid the bias introduced by a declarative approach or the presentation of specific cases.

\section{Methods}

\section{Study design}

The AIR II study was conducted between November 1997-April 1998. It followed a preliminary survey, AIR I, aimed at testing various collection methods in several French regions [9]. GPs $(n=2,000)$ were randomly selected and asked to participate in each of 30 predefined areas covering mainland France. The goal was to obtain a representative sample of at least 200 GPs per area. The objective of $10 \%$ of GPs participating in the study from the randomly selected $2,000 \mathrm{GPs}$ in the sample was only achieved or exceeded in six of the 30 regions, the other 24 regions providing only $3.5-9.1 \%$. However, if these regions are pooled in terms of geographical proximity into the following major regions: North, Brittany, SouthWest, South-East, Centre, North-East and Ile de France, a more homogeneous distribution is obtained with $5.6-9.2 \%$ of GPs in the sample in each of these seven major regions. The practice locations (rural and semi-rural or town $<30,000$ or $>30,000$ inhabitants) were each represented by approximately one-third of the medical population without any bias in terms of the GPs' age and sex. Their demographical data, year of qualification and the characteristics of their practice were obtained by means of a telephone call from the data processing centre. The GPs' participation was voluntary.

Each GP had to include the first two patients diagnosed as suffering from LRTI and being seen for the first time for the current episode, aged between 15-65 yrs and free from cancer, bronchiectasis, blood disorder or tuberculosis. They had to be human immunodeficiency virus-negative. Pregnant females were also excluded.

The patient's sociomedical record was completed by the GP during the consultation and sent to the data processing centre at the same time as an anonymous copy of his prescription. The GP also had to report the inclusion by telephone and agree to a telephone appointment with an interviewer. A telephone questionnaire enabled the investigator to collect specific information about the patient, the supplementary procedures decided upon by the GP and the reason for his choices. The interview was conducted on the basis of closed or multiple-choice questions (reason for consultation, diagnostic criteria, type of LRTI and auscultatory symptoms) without any prior recall of the symptoms usually associated with the diagnoses. It also included open questions on the prescription of investigations and treatment, work exemption or follow-up appointments, but not on risk or comorbidity factors. The characteristics of severity, such as respiratory or cardiac frequency, were not recorded since previous experience had shown that they were not accurately recorded during the consultation. Crackles were defined as focal signs on chest examination, in favour of pneumonia. Rhonchi represented the bronchial manifestation of sputum.

\section{Statistical analysis}

The GP and patient files were analysed separately. Descriptive analyses were undertaken on each form and were expressed as a percentage for qualitative variables and as a median for quantitative variables. Qualitative variables or those converted to qualitative variables were analysed with respect to explanatory data relating to the patients by bivariate analysis using Chi-squared or Fischer's test. A significance level of $5 \%$ was adopted.

\section{Results}

\section{General practitioners}

GPs $(n=3,144)$ who included at least one patient suffering from LRTI participated in the survey. Demographical data are summarized in table 1 and compared with the national statistics from the National Health Insurance Fund (Caisse Nationale d'Assurance Maladie (CNAM), Statistiques Nationales, personal communication).

\section{Patients}

Patients' records $(n=5,998)$ were received, 99 of which contained no sociomedical data and 420 failed to meet the inclusion criteria; 5,469 records were therefore included in the analysis. Consultations accounted for $73.6 \%$ of contacts and $26.4 \%$ of visits. The patients' demographical data are presented in table $1,84.8 \%$ of patients had additional medical insurance and all had basic social insurance cover, involving the general scheme in $84.1 \%$ of cases, the agricultural scheme in $6.5 \%$ and the craftsmen and tradesmen's scheme in $4.2 \%$ (versus $85 \%, 10 \%$ and $5 \%$, respectively, according to national statistics).

Table 1.-Demographic data of general practitioners and patients

\begin{tabular}{lccc}
\hline & Male & Female & $\begin{array}{c}\text { Age yrs } \\
\text { (median) }\end{array}$ \\
\hline $\begin{array}{c}\text { General practitioners } \\
\text { AIR II }\end{array}$ & $2547(81)$ & $579(19)$ & 40 \\
$\quad$ NHI Fund & $47051(77.7)$ & $13446(22.3)$ & 41.7 \\
$\quad \begin{array}{c}\text { December } \\
\text { 31 1997 }\end{array}$ & & & \\
Patients & & & \\
AIR II & $2901(53)$ & $2568(47)$ & 45 \\
\hline
\end{tabular}

Data are presented as $\mathrm{n}(\%)$ unless otherwise stated. AIR: Analyse Infections Respiratoires; NHI: National Health Insurance. 
Table 2. - Reasons for consultations, patients' complaints, and prescriptions

Characteristics

\begin{tabular}{lr} 
Reason for consultation & \\
Rhinopharyngeal respiratory symptoms & $989(18.5)$ \\
Bronchopulmonary respiratory symptoms & $4478(79.9)$ \\
Infectious symptoms & $1073(20.0)$ \\
Constitutional symptoms & $819(15.3)$ \\
Patients' complaints & \\
Fever & $3592(67.1)$ \\
Dyspnoea & $1270(23.7)$ \\
Cough & $471(88)$ \\
Sputum & $2895(54.1)$ \\
Chest pain & $640(12)$ \\
Other & $230(4.3)$ \\
Auscultatory signs (n=4497) & \\
Rhonchi & $3663(81.5)$ \\
Wheezing & $1093(24.3)$ \\
Crackles & $733(16.3)$ \\
None & $730(16)$ \\
Prescriptions & \\
Referral to specialist opinion & $66(1.2)$ \\
Hospitalization & $29(0.5)$ \\
Supplementary investigations & $579(10.8)$ \\
Sick leave & $1312(24.5)$ \\
Follow-up appointment & $1330(24.8)$ \\
Antibiotic & $5165(96.5)$ \\
Coprescription & $5115(95.5)$ \\
\hline
\end{tabular}

Data are presented as $n(\%) .(n=5354)$.

The reasons for consultation, symptoms reported by patients and auscultatory findings are presented in table 2. A total of 214 cases diagnosed as nose infections were excluded so 5,354 records were included. Five complaints or combinations of complaints were present in $>5 \%$ of patients, with these groups together accounting for $53 \%$ of the population.

Among these, only three combinations of symptoms and auscultatory abnormalities represented the clinical presentation of $>5 \%$ of patients: cough and expectoration $(698=13 \%)$, fever, dyspnoea, rhonchi and wheezing $(536=10 \%)$ and fever, expectoration, dyspnoea and crackles $(400=7.5 \%)$. However, chest pain was more frequently significantly associated with pneumonia $(32.2 \%)$ than AECB $(11.1 \%)$ or AB (9.4\%). Dyspnoea and sputum were more frequently associated with AECB than pneumonia or AB.

\section{Practical consequences of the diagnoses}

The diagnoses made by the GPs immediately after the consultations were pneumonia in 516 cases $(9.6 \%)$, $\mathrm{AECB}$ in 799 cases $(14.9 \%)$ and $\mathrm{AB}$ in 3,881 cases $(72.5 \%)$. Finally, 223 cases $(4.2 \%)$ were diagnosed as nose infections and 160 cases $(3 \%)$ involved none of the previous diagnoses.

Auscultatory signs, whether alone or combined, were present with each of the three diagnoses. Rhonchi were significantly $(p<0.001)$ more frequent in $A B$ $(69.8 \%)$ and in AECB $(79.9 \%)$ than in pneumonia $(49.6 \%)$. Wheezing was significantly more frequent in AECB $(39.9 \%)$ than in pneumonia $(19 \%)$ or $A B$ $(17.4 \%)$. Crackles were significantly more frequent in pneumonia $(43.8 \%)$ than in other LRTI, being present in $16.3 \%$ of AECB cases and $9.6 \%$ of AB. Pneumonia was characterized by a higher frequency of crackles either alone or combined with rhonchi, but the latter were present only in $43.8 \%$ of these patients in total.

\section{Complementary examinations}

LRTIs seen at consultation or during visits by GPs led to few requests for specialist examinations, hospitalization or supplementary investigations (table 3). Requests for a specialist opinion involved a lung specialist in $84.8 \%$ of cases, and an ear, nose and throat specialist in $4.6 \%$. Complementary investigations were a chest radiograph in $90.8 \%$ of cases, a white blood cell count in $14.2 \%$, a C-reactive protein in $5.2 \%$, a sinus radiograph in $3.8 \%$, an erythrocyte sedimentation rate in $4.1 \%$ and a microbiological sputum examination in $1.9 \%$.

Table 3. - Prescriptions of antibiotics and coprescriptions by classes and diagnoses

\begin{tabular}{lccc}
\hline & Pneumonia & $\begin{array}{c}\text { Acute exacerbation of } \\
\text { chronic bronchitis }\end{array}$ & Acute bronchitis \\
\hline Subjects n & 496 & 762 & 3656 \\
Medications & $184(37.1)$ & $304(39.9)$ & $1637(44.8)^{*}$ \\
$\quad$ Macrolides & $180(6.3)$ & $192(25.2)$ & $1099(30.1)^{*}$ \\
Aminopenicillins & $71(14.3)^{*}$ & $87(11.4)$ & $293(8.1)$ \\
Aminopenicillins+ $\beta$ lactamase inhibitor & $29(5.8)$ & $57(7.5)$ & $379(10.4)^{*}$ \\
1st generation cephalosporins & $83(16.7)$ & $149(19.6)^{*}$ & $407(11.1)$ \\
2nd and 3rd generation cephalosporins & $40(8.1)$ & $76(10)^{*}$ & $219(16.0)$ \\
Synergystins & $15(3.0)$ & $43(5.6)^{*}$ & $64(1.8)$ \\
Quinolone & $5(1.0)$ & $17(2.2)$ & $70(1.9)$ \\
Others & $292(59.5)$ & $539(71.0)^{*}$ & $2329(62.7)$ \\
Coprescriptions & $225(45.8)$ & $246(32.5)$ & $1813(48.9)^{*}$ \\
Mucomodifiers & $93(18.9)$ & $202(26.6)^{*}$ & $456(12.3)$ \\
Nonsteroidal anti-inflammatory drugs & $122(24.8)$ & $143(18.8)$ & $1040(28.0)^{*}$ \\
Steroidal anti-inflammatory drugs & $21(4.3)$ & $116(15.3)^{*}$ & $194(5.2)$ \\
Antitussives & & & \\
Bronchodilators & & & \\
\hline
\end{tabular}

Data are presented as n (\%). ${ }^{*}$ : aminoglycosides, lincosamide, rifamycin, phenicols, cyclins, sulphonamides. ${ }^{*}: \mathrm{p}<0.05$. 


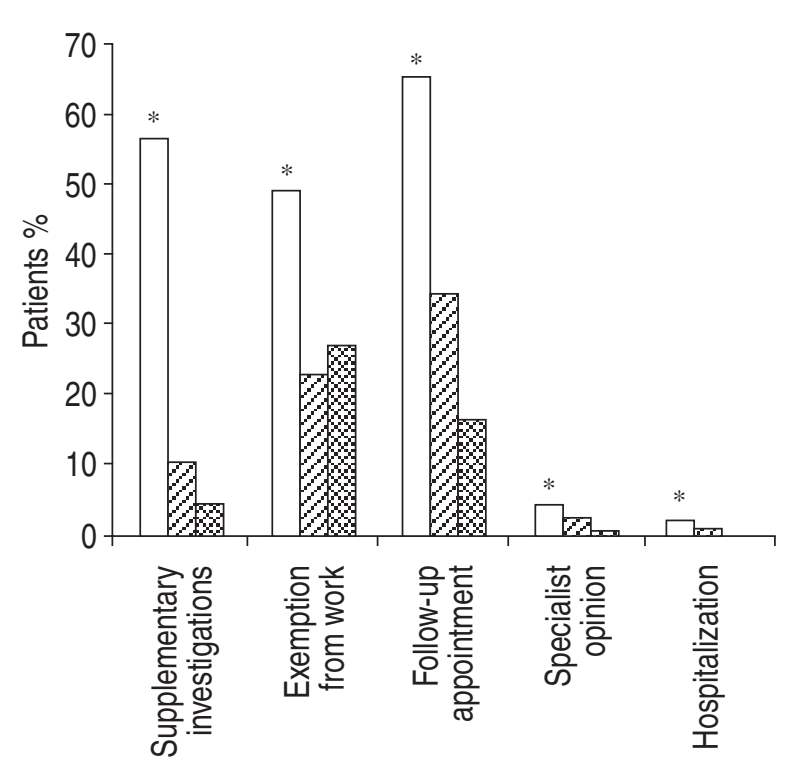

Fig. 1.-Complementary practice according to diagnosis. $\square$ : pneumonia; $\mathbb{Z}$ : acute exacerbation of chronic bronchitis; : acute bronchitis. *: $\mathrm{p}<0.05$.

Bivariate analysis confirmed a significant relationship between the diagnosis and the request for a specialist opinion, hospitalization, prescription of supplementary investigations, follow-up appointment and exemption from work $(\mathrm{p}<0.001)$, with all these prescriptions being relatively more frequent in pneumonia (fig. 1). Conversely, sociomedical parameters such as sex, occupation, having additional insurance or not, and region and place of practice had no effect on requests for complementary examinations.

\section{Antibiotic prescriptions}

A total of 5,270 patients were prescribed an antibiotic out of the 5,354 cases included in the analysis, i.e. $96.5 \%$ on average. Patients aged $<45$ yrs were prescribed an antibiotic in $95.6 \%$ of cases, versus $97.3 \%$ of those aged $>45$ yrs $(p=0.0007)$. The decision to hospitalize, although rare, was frequently not accompanied by an antibiotic prescription (79.3\% prescriptions versus $96.5 \%$ for non-hospitalized subjects; $\mathrm{p}=0.0001)$. The effect of the diagnosis also remained limited: an antibiotic was prescribed for pneumonia in $98.6 \%$ of cases and for $\mathrm{AB}$ in $96.5 \%$ $(\mathrm{p}=0.0001)$. However, prescriptions of antibiotics by class and diagnosis revealed statistically significant qualitative differences $(\mathrm{p}=0.0001$; table 3$)$. GPs reported that they prescribed antibiotics without any pressure from patients. Only $2.1 \%$ of GPs who did not prescribe antibiotics justified their choice because of recommendations.

\section{Coprescriptions}

Multiple coprescriptions are commonplace $(10,027$ prescriptions for 5,115 patients). The main nonantibiotic prescriptions are presented in table 3 versus diagnosis. In total, almost two-thirds of patients were prescribed a mucomodifier. Steroidal antiinflammatory drugs and bronchodilators were particularly prescribed in AECB, whereas antitussives were slightly less common.

\section{Discussion}

LRTI is one of the major reasons for antibiotic treatment. Many of these prescriptions are considered unjustified, particularly in the case of a presumed viral aetiology of acute bronchitis [10], or because the micro-organisms usually involved may not be sensitive to them. The gap between recommendations and practice has often been highlighted $[6,11]$. The aim of the present study was to evaluate the diagnostic process and prescriptions of GPs faced with an LRTI. The results show that these GPs: 1) encounter relatively mild infections, 2) do not perform additional examinations, and 3) have a high prescription rate for both antibiotic and symptomatic treatment.

This study represents a new step in analysing these prescriptions and in attempting to understand the reasons for them by placing them in their symptomatological and sociomedical context. Its originality lies in the fact that symptoms, supplementary diagnostic procedures, treatment (in terms of both antibiotics and coprescriptions) and sociomedical parameters most likely to influence prescriptions, were collected for the same patients. In addition, these details were recorded in real time from authentic cases to prevent the bias introduced by declarative or retrospective studies. GPs $(n=3,144)$ agreed to take part voluntarily and 5,469 cases were analysed. The initial calculation of the sample necessary for a representative study was based on a frequency of antibiotic prescriptions in $80 \%$ of cases, a figure which was exceeded. It also aimed to achieve a homogeneous distribution of the population of GPs across the seven predefined areas. This objective was not reached for all these areas. However, the distribution of GPs and patients was relatively homogeneous throughout the country. In addition, the comparison between the sample of GPs and the medical population, as listed by the National Health Insurance, means that this medical population is probably representative on a national scale. Likewise, in terms of the patients, their demographical data and the respective proportions of the different health insurance funds, which were very similar to the national data, were also indicative of their representativeness. In France, there is no financial advantage for the GP to ask for a referral or investigation, but this is not the case for reconsultation. It cannot be ruled out that the first two LRTI patients consulting their GP were not in fact included, so a selection bias is possible. However, this is the case in all studies using this kind of methodology [7]. Moreover, because the aim of the study was to evaluate the GPs' approach pragmatically, it is thought that this bias, if any, would only have a small influence on the results.

Patients consulted their GPs for bronchopulmonary respiratory symptoms. Fever was present in two-thirds of cases and patients' complaints were 
essentially isolated cough and expectoration. This has already been reported in the study of MACFARLANE et al. [10]. Few patients included in the study were admitted to hospital, as was the case in the report by SCHABERG et al. [12]. Therefore, a preliminary conclusion from this study is the predominantly benign nature of the cases observed. By excluding patients aged $>65 \mathrm{yrs}$, the protocol of the study certainly contributed to highlighting this benign aspect. The consensus conference [4] focused mainly on optimizing the management of severe cases. The method adopted, by encouraging GPs to adapt their management to the symptoms of severity, therefore coincides with current consultation practice.

It is generally considered that it is difficult to obtain a definitive diagnosis of LRTI following a consultation without a supplementary investigation, because of the poor sensitivity and specificity of the clinical symptoms [4]. The study methodology here, required the GPs to give an opinion on the diagnosis. This method was chosen to allow an assessment of the value of the symptoms and of the chest examination in guiding a presumptive diagnosis. Nevertheless, this diagnosis assumes very practical connotations, reflected in a highly differentiated approach to management in terms of supplementary investigations, specialist referrals, hospitalization and to a lesser extent, follow-up appointments and exemption from work. The present study, which is based on real-time collection of prescriptions, therefore gives a fairly different overview of the population of LRTI observed in general medical practice from that obtained by a previous large European declarative study [13], which estimated that, in France, $21 \%$ of patients underwent diagnostic investigations. In another declarative study, $100 \%$ of GPs stated that they would have performed a chest radiograph in a specific case of pneumonia in a 40-yr-old adult without risk factors, whereas in fact the figure was $54 \%$ [14].

Recommendations have been made to reduce or improve the prescription of antibiotics $[11,15]$. Nevertheless, the results are similar in terms of antibiotic prescription in this study and the previous European one [7]. Antibiotics were not given to $3.6 \%$ of patients compared to $17 \%$ in the European study. Nevertheless, a graduated approach can be observed in the issue of prescriptions, which takes into account the diagnosis of severity: macrolides are relatively less frequently prescribed, while aminopenicillins with a $\beta$-lactamase inhibitor and second and third generation cephalosporins are prescribed relatively more in pneumonia than in $\mathrm{AB}$. It has been indicated that the difference between the declared intention and the actual prescriptions suggests that GPs are very aware of the "right answer", i.e. the one they most often provide in declarative studies. This concept was already established by the previous study, which reported that GPs considered antibiotic therapy unnecessary in $51 \%$ of cases in which they had prescribed it for an LRTI [16]. Recently, it has been suggested that $50 \%$ of patients have direct or indirect evidence of infection, but the outcome seems to be unrelated to the identified pathogens [10]. This study casts new light on this prescription by reporting the relative proportion of antibiotics and coprescriptions.

The prescriptions of analgesics and/or antipyretics, antitussives, mucomodifiers and corticosteroids (or bronchodilators) accounted for two-thirds of all prescriptions. Each patient received $\geqslant 1$ of these, medications as often as antibiotics. All this suggests that the requirement made on the GP when consulted for a lower respiratory tract infection is to alleviate all the complaints immediately and to shorten the course of the disease by antibiotic therapy. In conclusion, this study confirms that consultations for lower respiratory tract infections are very common and mostly concern acute bronchitis. General practitioners saw relatively mild infections. They had a high rate of antibiotic prescriptions and symptomatic treatment. Efforts must be made to inform general practitioners about the management of lower respiratory tract infections, and to disseminate guidelines or published recommendations. This in turn raises the problem of how such information may be efficiently diffused to general practitioners in order to change their behaviour.

Acknowledgements. The authors are grateful
to F. Elkirk for assistance in preparing the
manuscript and to R. Cooke for assistance
with the manuscript.

\section{References}

1. Schwartz B. Preventing the spread of antimicrobial resistance among bacterial respiratory pathogens in industrialized countries: the case for judicious antimicrobial use. Clin Infect Dis 1999; 28: 211-213.

2. Seppald H, Klaukka T, Vtiopio-Varkila J, et al. The effect of changes in the consumption of macrolide antibiotics on erythromycin resistance in group A streptococei in Finland. N Engl J Med 1997; 337: 441446.

3. Guillemot D, Maison P, Carbon C, et al. Trends in antimicrobial drug use in the Community - France, 1981-1992. J Inter Dis 1998; 177: 497-500.

4. ERS Task Force Report. Guidelines for management of adult community-acquired lower respiratory tract infections. Eur Respir J 1998; 11: 986-991.

5. Arrêté du 13 novembre 1998 portant règlement conventionnel minimal applicable aux medecins en l'absence de convention médicale. Journal Officiel de la République Française 1998; 17147-17164.

6. Choutet $\mathrm{P}$, Crochet $\mathrm{B}$, Chaliwakilian $\mathrm{P}$, et al. Impact des références médicales opposables (RMO) sur la prescription des antibiotiques. Med Mal Infect 2000; 30: Suppl. 3, 185-192.

7. Huchon GJ, Gialdrom-Grassi G, Léophonte P, Manresa F, Schaberg T, Woodhead M. Initial antibiotic therapy for lower respiratory tract infection in the community: a European survey. Eur Respir J 1996; 9: 1590-1595.

8. Mulrow C, Cook D, Davidoff F. Systematic reviews; critical links in the great chain of evidence. Ann Intern Med 1997; 126: 389-391.

9. Taytard A, Daures JP, Arsac P, et al. AIR I. Analyse de la prise en charge des infections respiratoires basses 
en médecine générale en France. Rev Mal Respir 2001; 18: $163-170$.

10. Macfarlane J, Holmes W, Gard P, et al. Prospective study of the incidence, aetiology and outcome of adult lower respiratory tract illness in the community. Thorax 2001; 56: 109-114.

11. Agence du iriddicament. Prescription et consommation des antibiotiques en ambulatoire. Le concours médical 1999; 121: 760-768.

12. Schaberg T, Gialdroni-Grassi G, Huchon G, Leophonte P, Manresa F, Woodhead M. An analysis of decisions by European general practitioners to admit to hospital patients with lower respiratory tract infections. The European Study Group of Community Acquired Pneumonia (ESOCAP) of the European Respiratory Society. Thorax 1996; 51: 1017-1022.

13. Woodhead M, Gialdroni-Grassi G, Huchon GJ,
Léophonte P, Manresa F, Schaberg T. Use of investigations in lower respiratory tract infection in the community: a European survey. Eur Respir J 1996; 9: 1596-1600.

14. Vernejoux JM, Raherison C, Tunon de Lara JM, Taytard A. Evolution de la prise en charge des pneumopathies en médecine générale A Bordeaux (1992-1995). Rev Mal Respir 1999; 16: 65-70.

15. Schlemmer B, Trémolières F. Le rôle de l'Afssaps, de l'autorisation de mise sur le marché aux recommandations. Med Mal Infect 2000; 30: Suppl. 3, 193-196.

16. Macfarlane J, Lewis SA, Macfarlane R, Holmes W. Contemporary use of antibiotics in 1089 adults presenting with acute lower respiratory tract illness in general, practice in the UK: implications for developing management guidelines. Respir Med 1997; 91: 427-434. 